\title{
The influence of subcortical shortcuts on disordered sensory and cognitive processing
}

\author{
Jessica McFadyen ${ }^{\star 1,2,3}$, Raymond J. Dolan ${ }^{1,2}$ \& Marta I. Garrido $3,4,5,6$ \\ ${ }^{1}$ Max Planck UCL Centre for Computational Psychiatry and Ageing Research, London, UK. \\ ${ }^{2}$ The Wellcome Trust Centre for Neuroimaging, UCL, London, UK. \\ ${ }^{3}$ Queensland Brain Institute, University of Queensland, Brisbane, Australia. \\ ${ }^{4}$ Australian Research Council Centre of Excellence for Integrative Brain Function, Melbourne, Australia. \\ ${ }^{5}$ Melbourne School of Psychological Sciences, University of Melbourne, Melbourne, Australia. \\ ${ }^{6}$ Centre for Advanced Imaging, University of Queensland, Brisbane, Australia. \\ *j.mcfadyen@ucl.ac.uk
}

\section{Abstract}

The very earliest stages of sensory processing have the potential to alter how we perceive and respond to our environment. These initial processing circuits can incorporate subcortical regions, such as the thalamus and brainstem nuclei, which mediate complex interactions with the brain's cortical processing hierarchy. These subcortical pathways, many of which we share with other animals, are not merely vestigial but appear to function as 'shortcuts' that ensure processing efficiency and preservation of vital life-preserving functions, such as harm avoidance, adaptive social interactions and efficient decision-making. Here, we propose that functional interactions between these higher-order and lower-order brain areas contribute to atypical sensory and cognitive processing that characterizes numerous neuropsychiatric disorders.

\section{Introduction}

The human neocortex has undergone an evolutionary development beyond that of any other species, endowing us with a prodigious, albeit computationally demanding, facility for foresight, planning and abstract thinking. Even in situations of relative stability, there is often a need for immediate, rapid and adaptive responses. For example, when we accidentally touch a hot baking tray, our muscles will reflexively contract to protect our hand from danger even before we have felt an actual sensation of heat. This example of a biological shortcut enables us to perform time-precious actions, such as harm avoidance, by bypassing slower and more complex systems, such as those that involve strategic planning.

Neural shortcuts that stem from subcortical areas (which are the first to receive sensory input about the outside world ${ }^{1}$ ) can dynamically exert influence over and, in turn, be influenced by higher-order brain networks, to produce unique and intricate functional interactions. Over the past two to three decades, researchers have uncovered the structural properties of numerous subcortical shortcuts and their functional interactions with other circuits. Despite the potentially pivotal contribution of these dynamics to whole-brain processing, these lower-level circuits are underemphasized in models of complex sensory or cognitive processing, including models of disrupted information processing in psychiatric disorders. Here, we review recent progress in human and animal neuroscience on the anatomical and functional characteristics of putative rapid subcortical circuits. We propose a model of sensory processing that emphasizes the explanatory power of early subcortical processing and reciprocal subcorticalcortical interactions in generating and modifying behaviour and high-level cognition, and how this might contribute to aberrant processing in neuropsychiatric disorders.

\section{Rapid responses to threat}

The most intuitive example of recruitment of a neural shortcut is when we are faced with a potential threat. The faster we can perceptually discriminate between, say, a snake and a stick, the sooner we can initiate a potentially life-saving fight-or-flight response. Overexertion or underexertion of this ability can severely impact on quality of 
life. For example, people with clinical anxiety, such as specific phobia, exhibit extreme fear and hastened detection of relatively innocuous stimuli (for example, a photograph of a spider) ${ }^{2}$. On the other end of the spectrum, people with psychopathy have diminished threat detection and responsivity, which may contribute to antisocial behaviour ${ }^{3}$. This raises a question of how sensory information first reaches neural regions that elicit crucial, expedited responses to threats, and how might this be changed in people with symptomatically altered threat processing.

\section{The subcortical route to the amygdala}

In the 1980s, researchers discovered that rodents possess a neural shortcut that connects early auditory processing regions within the brainstem to the amygdala via the thalamus. This so-called 'subcortical route to the amygdala' (or 'innate alarm system') effectively bypasses the auditory cortex, transmitting auditory information directly from the thalamus to the amygdala and triggering conditioned fear responses such as freezing and elevated heart rate 4 . An equivalent auditory or visual pathway in the human brain would suggest that the amygdala could more readily initiate behavioural responses to threat by using rapid, but crude, sensory input from the subcortical route, before receiving a more refined sensory input provided by cortical processing streams (see Fig. 1). This proposition has been a subject of intense debate ${ }^{5,6,7,8}$. One source of this controversy has arisen out of the unique challenge posed by studying fast activity in human subcortical regions in vivo. Significant advances in computational modelling of neuroimaging data ${ }^{9}$, along with a growth of relevant animal research ${ }^{10}$, have helped enable investigation into these subcortical dynamics.

\section{[FIGURE 1]}

Figure 1. Analogous neural networks for threat responses across species. Diagrams of basic visual threat response networks for the rodent (left) and human (right) brain. The transmission of visual information from the retina to threat-related regions (for example, the amygdala (AMG) and striatum (ST)) is similar across species, although the dominance of vision in humans has meant an increase in the size of the pulvinar (PUL; rodent equivalent is the lateral posterior nucleus of the thalamus (LP)) and visual cortices ${ }^{39}$. a | The superior colliculus (SC) in rodents receives the majority of retinal input ${ }^{19}$ and projects to the parabigeminal nucleus (PBN), a small satellite nucleus ${ }^{15}$. b | In contrast, in humans, the majority of retinal input is received by the lateral geniculate nucleus $(\mathrm{LGN})^{165}$. c | Looming visual stimuli pose an evolutionary threat to rodents, as they signify the presence of a flying predator. d | In humans, visual threat cues are distinguished by more complex information, such as animal categories (for example, snakes, spiders) and social cues (for example, fearful expression in conspecific). The cortical visual processing stream is indicated by blue arrows and subcortical processing streams are indicated by orange arrows. dIPFC, dorsolateral prefrontal cortex; ES, extrastriate cortex; IT, inferotemporal cortex; LES, lateral extrastriate cortex; mPFC, medial prefrontal cortex; PAG, periaqueductal gray; V1, primary visual cortex; vmPFC, ventromedial prefrontal cortex; VTA, ventral tegmental area.

The amygdala and other subcortical structures, such as the thalamus, provide examples of structures that are highly conserved across evolution ${ }^{11}$, and this allows informative comparisons between the human brain and the more accessible and manipulable brains of other species. Although rodents rely strongly on auditory and tactile stimuli, they also demonstrate freezing and escape behaviour to looming visual stimuli ${ }^{12}$ (as do humans ${ }^{13}$ ). Using optogenetics and neuroanatomical tracing, a visual pathway from the superior colliculus (SC) to the lateral posterior nucleus of the thalamus (LP), and thence to the amygdala, has been identified in mice ${ }^{14}$ (see Fig. 1). Additional paths have also been identified between the SC and the amygdala that traverse the parabigeminal nucleus (a small satellite nucleus of the SC) ${ }^{15}$, the ventral tegmental area ${ }^{16}$, the ventral midline thalamus ${ }^{17}$ and the periaqueductal grey (PAG) ${ }^{18}$.

Despite the fact that around $90 \%$ of retinal ganglion cells project to the SC in the rodent brain ${ }^{19}$, compared with about $10 \%$ in the primate brain ${ }^{20}$, researchers have recently traced an anatomical pathway from the SC to the amygdala in the macaque monkey brain ${ }^{10}$. This pathway has also been successfully reconstructed from diffusion imaging data in humans ${ }^{9,21}$ and non-human primates ${ }^{21,22}$. Thus, despite the considerable structural reorganization of the primate visual system compared with that of rodents, visual pathways from the SC to the amygdala appear to have been conserved through evolution across different species. In the following sections, we ask whether pathways emanating from the SC that bypass the visual cortex have a meaningful influence on behaviour. 


\section{Parallel paths for defensive behaviour}

In the rodent brain, SC-originating shortcuts have genetically identifiable cell types that causally and independently evoke distinct behavioural responses to visual signs of threat or prey. The SC-LP-amygdala pathway, which mediates freezing ${ }^{14}$ and prey detection ${ }^{23}$, receives input from retinal ganglion cells that prefer small, slow stimuli (for example, a distal flying predator), whereas the SC-parabigeminal nucleus-amygdala pathway, which triggers escape $^{15}$ and prey pursuit behaviour ${ }^{23}$, receives input from retinal ganglion cells that prefer large, fast stimuli (for example, a proximal collision $)^{24}$. A recent optogenetic study has demonstrated that silencing the mediating node of either pathway (that is, the LP or the parabigeminal nucleus) results in a dominance of the other path's behavioural output ${ }^{25}$. Altogether, these results from rodent research suggest that differences in the neurobiological architecture of parallel subcortical pathways support optimal dimorphic defensive behaviours from the earliest stages of visual processing.

Although the size and speed of a visual stimulus give some indication as to which defensive behaviour might be optimal, we also know that rapid decision-making under threat reflects a larger and more complicated parameter space. For example, in response to a looming visual stimulus, mice will switch from escape to freezing if they know that there is no accessible safe location to escape to ${ }^{26}$. If near a food source, crayfish will freeze instead of flee in response to a fast visual stimulus ${ }^{27}$. The exact mechanism by which contextual information is integrated with bottom-up subcortical processing has yet to be fully explored, although there are emerging hints in the literature that we discuss below ${ }^{28}$.

One possible integration mechanism is that the SC itself is primed with contextual information from other neural sources, so that incoming sensory information is biased towards or away from freezing or fleeing from the very beginning of processing. This is encapsulated by hierarchical predictive processing frameworks ${ }^{29}$, which we discuss in more detail below. Another possibility, and one that is more commonly considered by the current literature ${ }^{30}$, is that contextual information is integrated at a later stage, such as at the PAG or the amygdala, at which point the trajectory towards different defensive behaviours is altered. Below, we present evidence that supports a framework that considers both possibilities.

\section{Defensive behavioural decisions}

Activity within the SC is subject to modulatory influences of other subcortical and cortical regions. For example, GABAergic projections from the substantia nigra pars reticulata can inhibit the SC, significantly reducing threat recognition and increasing approach (as opposed to avoidant) behaviour ${ }^{31}$. The behavioural specificity of this upregulation or downregulation is thought to arise from the point at which a modulatory connection synapses onto the SC, either its lateral division (associated with approach behaviour) or its medial division (associated with avoidant behaviour). Indeed, if projections from the visual cortex to the SC (which synapse exclusively in the medial $\mathrm{SC}^{32}$ ) are silenced, the magnitude of freezing behaviour is reduced by approximately one-third ${ }^{33}$. In contrast, projections from the locus coeruleus (which synapse to both the medial and the lateral SC) accelerate escape decisions when rodents are stressed but can also attenuate escaping when stress is reduced via gentle handling ${ }^{34}$. We can speculate that, after recently learning that a safe escape location is now inaccessible, afferent connections from the hippocampus to the medial SC $^{32}$ might suppress escape behaviour by transmitting an inhibitory signal ${ }^{28}$.

Like rodents, pharmacological activation of the SC in non-human primates evokes defensive behaviours, such as freezing, escape, cowering and alarm vocalizations ${ }^{35,36}$. However, if the amygdala is also inactivated, only cowering behaviour is disrupted ${ }^{36}$. In contrast, pharmacological activation of the PAG in macaques produces vocalizations but not motor-related defensive responses, such as escape or cowering ${ }^{37}$. Together, these results support the finding that, in the rodent brain, the SC triggers escape behaviour in the PAG after neural gain ramps up a particular decision threshold ${ }^{18}$, similar to the established role of the SC in issuing motor commands for eye and head movement, and reaching in both rodents and primates ${ }^{38}$.

In comparison with rodents, the visual processing capabilities of the primate SC and pulvinar (which also receives retinal input ${ }^{39}$ ) have evolved to be considerably more sophisticated. For example, the SC and pulvinar in primates 
respond preferentially to images of snakes and emotional facial expressions at latencies of $25 \mathrm{~ms}$ (SC) and 50$60 \mathrm{~ms}$ (pulvinar) ${ }^{40,41,42}$ (see Box 1). Hence, when behavioural coordination regions, such as the amygdala, receive rapid subcortical input from areas such as the pulvinar, different visual representations are already selectively enhanced and may bias subsequent behavioural responses. Below, we explore further the computations made by subcortical visual areas and how these are influenced by, and/or exert influence over, cortical regions to influence perception, cognition and behaviour. From a starting point of threat processing, we expand the discussion to incorporate general perceptual decision-making and predictive processing, and draw key relationships to psychiatric pathology. 


\section{Box 1 | Functional response latencies in subcortical and cortical visual networks}

The implication of a subcortical 'shortcut' is that it enables some sort of functional processing or behaviour to occur more quickly than via canonical networks. Mapping out the precise temporal dynamics of human circuits is notoriously difficult, owing to variance caused by different stimuli, the size and morphology differences between animal (from which most direct latency information is acquired) and human brains, and the limited neural regions that can be simultaneously recorded. The visual system is one of the most thoroughly examined and below we summarize key latency findings to date.

In the primate brain, visual information from the retina initially perturbs the lateral geniculate nucleus $\left(\sim 20-30 \mathrm{~ms}\left(\right.\right.$ ref. $\left.\left.{ }^{173}\right)\right)$, followed by the primary visual cortex (V1; $40 \mathrm{~ms}\left(\right.$ refs $\left.^{169,174}\right)$ ) and then the superior colliculus (SC; 50 ms (refs $\left.{ }^{41,169}\right)$ ) and pulvinar $\left(\sim 60 \mathrm{~ms}\left(\right.\right.$ ref $\left.\left.^{55}\right)\right)$. Activity then spreads along the rest of the cortical visual stream, including visual area 3 (V3), the middle temporal area (V5), V2 and V4 (ref. ${ }^{175}$ ). The exact mean and earliest latencies vary widely across studies, however, depending on multiple factors (for example, whether the animals are awake or anaesthetized, the type of stimuli used and so forth).

The delayed firing of the SC, as compared with $\mathrm{V} 1$, might seem surprising given that it receives direct input from the retina ${ }^{20}$. However, there are some circumstances under which SC firing precedes that of V1. For example, the mean response latency of the SC shifts from 86 to $59 \mathrm{~ms}$ under higher stimulus intensity ${ }^{176}$. Even earlier shifts are seen in the SC to face-like patterns, which elicit neuronal responses in the SC after only $\sim 30 \mathrm{~ms}$ on average ${ }^{41}$. Similarly, the pulvinar also responds earlier to snakes $\left(\sim 55 \mathrm{~ms}\left(\right.\right.$ ref. $\left.\left.{ }^{40}\right)\right)$ and faces $\left(\sim 50 \mathrm{~ms}\left(\right.\right.$ ref. $\left.\left.{ }^{55}\right)\right)$ than other stimulus categories. These early neuronal responses to biologically relevant stimuli can explain findings for early $(\sim 75 \mathrm{~ms})$ innervation of the amygdala ${ }^{136}$ by the pulvinar ${ }^{138}$. In comparison, cortical processing routes to the amygdala have been computationally estimated to take about $145-170 \mathrm{~ms}\left(\right.$ ref. $\left.{ }^{177}\right)$.

The early responses to these stimulus categories in the SC and the pulvinar could relate to the computation of saliency maps. Visual properties (for example, luminance, contrast, edges, motion, colour and so forth) are converted into saliency maps (that is, the degree to which a point on a topographic map differs from its surroundings) and priority maps (that is, which region of a topographic map to allocate attention to, based on bottom-up saliency and top-down goals ${ }^{170}$. Saliency maps are rapidly encoded by V1 neurons after receiving bottom-up input. Saliency maps in the SC, however, precede those in V1 by approximately $50 \mathrm{~ms}\left(\right.$ ref. $\left.^{169}\right)$. Hence, visual stimuli are filtered at very early stages for aspects such as saliency and biological relevance.

\section{Subcortical impact on cognition}

Given the finite processing capabilities of the human brain, sensory information is continuously filtered to strengthen representations of the most relevant stimuli. This can occur via innate neurobiological properties, such as the preferential firing of the SC and the pulvinar to biologically relevant stimuli (for example, snakes, faces $)^{40,41,42}$, as well as via higher-order computations such as attentional allocation and predictive inference.

\section{Attentional biases}

Both the SC and the pulvinar are implicated in capturing and allocating attention. The SC directs eye movements and covert attention towards salient visual stimuli, and can do so independently of the visual cortex ${ }^{43}$. The pulvinar also plays a regulatory role in attention by synchronizing cortical activity according to attentional allocation ${ }^{44}$. Visual coding within the pulvinar itself reflects attentional allocation, with attention increasing the precision of a visual stimulus representation ${ }^{45}$. These computations allow the gain of different visual representations to be altered before being transmitted to higher-order regions, such as the amygdala or frontal cortices.

\section{Anxiety}

An attentional bias towards certain stimuli, and away from others, has implications for how an agent responds to, and learns from, their environment ${ }^{46}$. Anxiety disorders have generally been associated with a selective attentional bias towards threatening stimuli ${ }^{47}$. For example, people with spider phobia show heightened detection sensitivity, and lower decision thresholds, for spider images presented rapidly amongst a stream of other images ${ }^{2}$. Similarly, people with spider phobia overestimate the speed of spider stimuli moving towards them compared with away from them ${ }^{48}$ and also overestimate the size of real-life spiders ${ }^{49}$. Intriguingly, recent computational evidence suggests a bidirectional relationship between attention to threat and aversive learning, such that attentional biases towards threat coincide with enhanced learning about aversive stimuli46. 
A recent influx of human neuroimaging studies suggests a functional role of SC and pulvinar paths in biasing attention towards threat. Diffusion imaging studies have shown that people with greater fractional anisotropy along the SC-pulvinar-amygdala path also have a stronger bias in orienting towards negative images ${ }^{50}$ and are also better at recognizing fearful facial expressions ${ }^{9}$. The structural connectivity of this pathway also correlates with forward-flowing effective connectivity, suggesting a directional, causal relationship ${ }^{9}$. While viewing spiders, effective connectivity from the pulvinar to the amygdala is greater in people with spider phobia than in those without ${ }^{51}$. Similarly, effective connectivity between the pulvinar and the visual and frontal cortices is greater in people with social anxiety disorder while viewing faces ${ }^{52}$. Women with post-traumatic stress disorder (PTSD) (which is characterized by a hypervigilant attentional bias to threat) exhibit a greater blood oxygen level-dependent (BOLD) signal in the SC, PAG and locus coeruleus ${ }^{53}$, as well as enhanced functional connectivity between the SC and the cingulate cortex, insula and amygdala, while viewing faces ${ }^{54}$. Overall, these studies support a bottom-up exaggeration of threatening stimuli in people with greater attentional bias to threat, such as those with clinical anxiety.

Notably, the stimuli used in the studies discussed tend to be restricted to faces, spiders and snakes, to which the SC and pulvinar innately respond $40,41,42,55$. Further research is needed to elucidate whether there is a genetic explanation for a neural responsivity of SC and pulvinar pathways to evolutionary threats (indeed, animal and social phobias are $30-40 \%$ heritable $\left.{ }^{56}\right)$. Importantly, rodent research has shown that a build-up of firing activity in SC neurons (likely as a result of a loop between the SC, substantia nigra pars reticulata and basal ganglia ${ }^{57}$ ) is associated with successful avoidance of fear-conditioned stimuli, a finding that extends SC functions from innate threats to include learned threats ${ }^{58}$.

\section{Autism}

Autism is a highly diverse and heterogeneous spectrum of developmental disorders that are broadly characterized by impaired social interaction and communication. Numerous cognitive theories have been developed to explain the pathogenesis of autism, and these include low-level visual processing ${ }^{59}$ and mirror neurons ${ }^{60}$ through to predictive coding ${ }^{61}$. An SC theory of autism draws from the wealth of evidence for disrupted or altered SC functioning in attention and face processing, as well as parallels between neural visual development and the onset of autism.

Autism is associated with atypical responses in the SC, pulvinar and amygdala in response to faces ${ }^{62,63,64}$ and looming stimuli65. More indirectly, there are strong similarities between many of the core pathologies of autism and key functional roles of the SC, such as the dominance of local over global visual processing ${ }^{66}$ (which also occurs as a result of $\mathrm{SC}$ deactivation ${ }^{67}$ ) and disrupted multisensory integration ${ }^{68}$ (which develops in neurons of the $\mathrm{SC}^{69}$ ). Similarly, the SC develops earlier than geniculostriate visual processing streams and thus dominates visual processing during the same period in which autism manifests (from 2 months to 3 years old) ${ }^{70}$. Overall, the similarities between SC development and function and the aetiology and current theoretical accounts of autism point to a prominent role of the SC, and perhaps also the pulvinar and lateral geniculate nucleus, in early visual computation ${ }^{59}$.

\section{Attention-deficit hyperactivity disorder}

The SC has long been implicated in sensory processing characteristics of attention-deficit hyperactivity disorder $(A D H D)^{71}$. There are two primary drivers of this hypothesis. The first is that people with ADHD have difficulty making the types of saccades specifically related to SC functioning ${ }^{72}$ - for example, anti-saccades (looking away from a target ${ }^{73}$ ) and express or micro saccades (saccades with very short latency ${ }^{74}$ ). The second driver is that the $\mathrm{SC}$ is a target of therapeutic action by amphetamines and methylphenidate, which relieve people with ADHD from distractibility and increase sustained attention. Rodent models of ADHD have indicated that these drugs dampen overall hyperactivity ${ }^{75,76}$ and increase the signal-to-noise ratio in the $\mathrm{SC}^{77}$. Furthermore, inhibitory connections from the dorsolateral prefrontal cortex to the SC are thought to prevent distractibility ${ }^{78}$.

These findings suggest that a pathology within the SC may result in it inaccurately computing saliency maps, such that more stimuli are perceived as maximally salient. This in turn might arise from the substantia nigra, which is 
known to relay signals for the reward value of visual stimuli from the caudate tail to the SC to inhibit saccades to irrelevant, low-value stimuli and vice versa ${ }^{79}$. Indeed, reward-evoked activity in the striatum (which projects to the substantia nigra) is reduced in children with $\mathrm{ADHD}^{80}$. Interestingly, the pulvinar (which projects to the striatum ${ }^{81}$ ) has reduced volume ${ }^{82}$ and abnormal functional connectivity with areas, including the prefrontal cortex ${ }^{83,84}$ and striatum ${ }^{84}$, in people with ADHD. This dynamic subcortical circuit, consisting of the SC, pulvinar and basal ganglia, may play a crucial role in pathological distractibility.

\section{Pre-attentive and unconscious biases}

\section{Blindsight and spatial neglect}

A powerful demonstration of subcortical influences on cognition is seen in the instance of blindsight. Blindsight describes a remarkable phenomenon whereby people who are cortically blind (that is, lesions to the primary visual cortex (V1) prevent conscious visual experience) can still respond to visual stimuli (see Box 2). Residual abilities include discrimination between stimulus categories (for example, lines versus no lines ${ }^{85}$, neutral versus fearful face ${ }^{86}$ ) as well as an ability to respond physiologically (for example, pupil dilation) to emotional stimuli87. Patients with blindsight can even navigate an obstacle course, despite being unable to report how they are able to do so ${ }^{88}$. Blindsight highlights two important features of the brain: first that $\mathrm{V} 1$ is an important neural substrate for visual consciousness $^{89}$ (but note that visual experience can be realized if V1 lesions occur in very early-life stages ${ }^{90}$ ); and secondly, neural networks that bypass V1 support a range of residual visual capacities that aid preservation of critical behavioural responses to visual stimuli.

Unconscious responses to affective stimuli are subserved by the SC-pulvinar-amygdala pathway, both in blindsight and in healthy people whose conscious visual perception is suppressed via experimental techniques (for example, backward masking, continuous flash suppression) ${ }^{7}$. In a particularly compelling case study of a patient with unilateral V1 damage, researchers found that fractional anisotropy increased along the SC-pulvinaramygdala path in the hemisphere with the $\mathrm{V} 1$ damage alone ${ }^{22}$. This indicates a possible neuroplastic, compensatory change along this neural pathway as a result of the patient's reliance on unconscious visual processing. A similar strengthening is seen along the pulvinar's projections to the motion-sensitive middle temporal area (V5) in marmosets who sustained early-life V1 lesions ${ }^{39}$. Indeed, both the SC and the pulvinar ${ }^{91}$ (as well as the lateral geniculate nucleus ${ }^{92}$ ) subserve residual motion processing in blindsight. In both affective and motion blindsight, there is likely a fundamental role of the SC in computing and transmitting saliency maps ${ }^{93}$.

People may also be 'blind' to stimuli in the left or right side of space owing to cortical lesions (most commonly the right parietal cortex). This is known as spatial neglect (or visual extinction), a condition where patients unknowingly ignore the contralesional side of space. The deficits in saliency encoding and spatial attention arise from lesioninduced disruptions to dorsal and ventral frontoparietal networks ${ }^{94}$. Included here are connections between the frontal eye fields and the SC, which are diminished in patients with spatial neglect and correlate with more impaired exploratory saccade behaviour ${ }^{95}$ (see Fig. 2). Despite this, patients with spatial neglect can retain residual, implicit, visual processing of unattended stimuli96, much as seen in patients with blindsight with $\mathrm{V} 1$ lesions. This observation demonstrates that residual visual processes are retained even after large-scale neural network disruptions, perhaps preserved by alternative pathways.

\section{[FIGURE 2]}

Figure 2. Interactions between cortical and subcortical networks during attentional allocation. The superior colliculus (SC) is a layered structure, consisting of superficial layers that receive retinal input and input from striate and extrastriate cortices $^{166}$, intermediate layers that receive input from the frontal eye fields (FEF) and are involved in saccade generation ${ }^{167}$, and deep layers that combine multisensory input to produce motor commands ${ }^{168}$. Neurons within the SC encode saliency maps in retinotopic space (see top left) earlier than the primary visual cortex (V1) ${ }^{169}$. For example, in a display of three simple stimuli (a red triangle, blue circle and blue triangle, as depicted bottom left), red may be encoded as more salient than blue, creating a pop-out effect of the red triangle. These saliency maps are combined with priority maps (that is, to where attention should be allocated, according to an attentional set for certain visual stimuli), which are created by higher-order regions and transmitted to the SC via top-down connections from areas, such as the FEF ${ }^{170}$. For example, the pop-out effect of a prominent colour may be overridden by an attentional set for a certain shape, such as when searching for a circle amidst triangles (bottom left). 
Saliency and priority maps are propagated throughout the brain but, in the case of blindsight, the absence of V1 places this ability solely with the SC and its connections to the pulvinar (PUL) ${ }^{7}$. The disruptions to FEF seen in spatial neglect could, in contrast, remove any early top-down influence on visual attention allocation ${ }^{94}$. The PUL reciprocally connects to multiple networks, some of which are displayed here (for example, the amygdala (AMG), orbital frontal cortex (OFC), dorsolateral prefrontal cortex (dIPFC), striatum (ST) and so forth) ${ }^{39}$. The PUL has been discovered to synchronize local field potentials across cortical networks, specifically visual area 4 (V4) and the inferotemporal cortex (IT), according to attention allocation ${ }^{44}$ (see top right). V5, middle temporal area.

\section{Box 2 | Subcortical contributions to blindsight}

The neural circuitry enabling blindsight, particularly for motion, has been thoroughly explored. Research has revealed the lateral geniculate nucleus (LGN), superior colliculus (SC), pulvinar, amygdala and extrastriate cortex (notably, the motionsensitive visual middle temporal area (V5) that is the earliest cortical region to receive visual input ${ }^{178}$ ) as key components ${ }^{179}$. However, there are considerable discrepancies in relation to whether the LGN or, alternatively, the SC and/or pulvinar subserve blindsight via connections to V5.

In support of a role for the LGN, there are reports of enhanced structural connectivity ${ }^{180}$ and functional connectivity ${ }^{181}$ between the LGN and V5 in patients who are cortically blind who have motion-related blindsight, whereas connectivity between the pulvinar and $\mathrm{V} 5$ is the same irrespective of whether or not a patient has blindsight ${ }^{181}$. Furthermore, residual neurons within the macaque LGN (which becomes profoundly degenerated after V1 lesions) transmit visual information to V5 that support preserved visual abilities ${ }^{92}$, regardless of in which life stage a V1 lesion is incurred ${ }^{165}$.

In support of the SC and the pulvinar, macaques with early-life V1 lesions show strengthened structural connections between the pulvinar and V5 (ref. $\left.{ }^{39}\right)$. Patients with blindsight show a greater blood oxygen level-dependent signal response in the SC and pulvinar during unconscious motion detection ${ }^{182}$. In line with this, patients with pulvinar lesions also tend not to have blindsight ${ }^{183}$. Similarly, V1-lesioned macaques cannot make visually guided saccades in the blind visual field if the connection between the $\mathrm{SC}$ and the pulvinar is pharmacologically inhibited ${ }^{184}$.

Without a direct test comparing the effects of LGN with SC/pulvinar inhibition on blindsight, we cannot definitively conclude whether one or both structures are critical to compensatory visual networks subserving blindsight. Nonetheless, it is thought that both play a role and that their relative contributions depend on multiple factors, such as the nature of the visual stimulus $^{184,185}$ as well as the developmental stage in which a V1 lesion is acquired. Tectopulvinar pathways develop earlier than geniculostriate pathways and so pulvinar-dominant networks tend to subserve blindsight in cases where $\mathrm{V} 1$ lesions are acquired earlier in life ${ }^{22,90}$ (but note that the late patient T.N. acquired V1 lesions at the age of 52 years and yet had markedly enhanced neural activity in the superior colliculus and pulvinar $\left.{ }^{86,88,137}\right)$.

\section{Implicit neural processing}

Pre-attentive or unconscious processing in spatial neglect and blindsight impacts conscious perception and decision-making ${ }^{7}$. For example, patients with spatial neglect can more easily semantically categorize an image (that is, identifying and naming the stimulus as it becomes progressively less blurry) when, in a previous session, this same image had been implicitly presented to the blind field of view. Patients with blindsight with V1 lesions show significantly faster perceptual discrimination (for example, the orientation of Gabor patches) of consciously perceived stimuli when fearful faces are concurrently and unknowingly presented in the blind field ${ }^{97}$. Similarly, facial expression recognition is improved when congruent emotional expressions are simultaneously presented to the blind hemifield in patients with $\mathrm{V} 1$ lesions $^{98}$ and in healthy people using conscious suppression techniques ${ }^{99}$ (but note that studies have also found facilitation by incongruent emotion pairs ${ }^{100,101}$ ). These congruency effects coincide with a greater BOLD signal in the SC, amygdala and fusiform gyrus ${ }^{98}$.

Studies in healthy populations using methods such as continuous flash suppression have also shown that perceptual evidence can still be accumulated from unconsciously presented moving stimuli (for example, the direction of motion). The rate of evidence accumulation enhances responses to subsequent consciously presented motion stimuli, such that accuracy for detecting the motion direction of the conscious stimulus is higher if the unconscious motion was congruent ${ }^{102}$. This has no effect on subjective confidence estimates and can even be trained ${ }^{103}$. Finally, fearful faces ${ }^{104}$ and fear-conditioned visual features ${ }^{105}$ that are initially suppressed from awareness using continuous flash suppression tend to 'break through' into perceptual awareness earlier than other 
neutral stimuli. Altogether, these studies exemplify the influence of non-conscious processing on subsequent conscious processing.

\section{Implicit processing in psychiatry}

Evidence for residual visual processing in blindsight and spatial neglect begs the question of whether nonconscious visual processing in subcortical networks contributes to disordered perception, memory formation or decision-making in psychiatric disorders. There is indeed evidence for exaggerated subcortical responses to consciously imperceptible stimuli in certain psychopathologies. For example, hyperactivity in the basolateral amygdala in response to subliminal fearful faces has been reported in people with higher trait anxiety ${ }^{106}$, with spider phobia ${ }^{107}$ and, to an even greater degree, with PTSD ${ }^{108}$. In contrast, people with autism have reduced amygdala activation in response to subliminally presented faces ${ }^{109}$ and also make significantly more saccades towards subliminal face stimuli with averted, rather than direct, gaze ${ }^{110,111}$. This suggests that neural circuits mediating unconscious visual responses may be hyper-responsive to direct gaze in autism, resulting in avoidance (for example, attentional disengagement).

\section{Predictive biases}

The content of our conscious experience reflects in, large part, our current model of the world ${ }^{112}$. In cases where sensory input is noisy or unreliable (due to either external factors, such as lighting conditions, or internal factors, such as impaired attentional filtering of incoming sensory information), our posterior estimate of the world becomes biased towards more precise prior expectations. Extremely precise priors (for example, over-expectancy of threat in anxiety disorders ${ }^{113}$ ) may exaggerate this even further. A reliance on prior expectations can profoundly shape perceptual experience, resulting in phenomena such as illusory percepts ${ }^{114}$ through to an accelerated entry of expected stimuli into conscious awareness ${ }^{115,116}$. Hence, the way that we consciously perceive ambiguous information can provide insight into our top-down predictions and a priori biases (see Fig. 3).

\section{[FIGURE 3]}

Figure 3. Updating of beliefs using sensory evidence and prior expectations. Within a hierarchical predictive processing framework, our beliefs are influenced by both the likelihood and prior expectations for a certain hypothesis ${ }^{29}$. In this example, the two competing hypotheses are that a visual stimulus is a neutral face or a fearful face (category evidence is represented by the $\mathrm{x}$ axis of each graph). a | The likelihood probability distribution (top; corresponding to the sensory evidence) favours a neutral face but is imprecise (for example, the room is dark and it is difficult to see). The prior probability distribution, however, is heavily biased towards a fearful face and is very precise, such as might be seen in clinical anxiety. Below this, the transmission of top-down predictions and bottom-up prediction errors is shown between neural regions. Aversive prediction errors are found in areas such as the amygdala ${ }^{130}$ and striatum (ST) ${ }^{132}$, and are thought to likely occur in the pulvinar (PUL) ${ }^{1}$. This suggests that early sensory processing at the level of the superior colliculus (SC) and lateral geniculate nucleus (the latter not shown) is not influenced by prior expectations. The rapid processing subserved by connections such as the SC-PULamygdala pathway, however, suggests that aversive prediction errors (that is, from prefrontal cortex (PFC) to amygdala) may be generated more quickly, which could constitute a key function of this subcortical circuit. The red arrows represent enhanced prediction errors and the blue arrows represent top-down threat expectations. b | In contrast, this example depicts a situation whereby the observer may not know what to expect (that is, an imprecise prior midway between the two hypotheses) but sensory evidence is biased towards a fearful face, producing the same biased belief (that is, similar posterior distribution) as the first example. Here, the biasing of sensory evidence towards threat may be subserved by multiple processes. First, signs of stress or hyperarousal (for example, fast heart rate) signalled by the periaqueductal grey (PAG) ${ }^{171}$ may be incorporated as sensory evidence of threat ${ }^{142}$. Secondly, the innate responsivity of the SC and PUL to fearful faces ${ }^{41,139}$ may exaggerate any low-level visual features congruent with fearful faces. Thirdly, the imprecise prior expectations from areas such as the dorsomedial prefrontal cortex (dmPFC) ${ }^{172}$ enhance the influence of the likelihood over the posterior estimate (that is, the belief). Hence, this demonstrates two different examples by which top-down predictions interact with sensory processing in subcortical circuits to give rise to biased beliefs in disorders such as anxiety, autism and schizophrenia. The red arrows represent enhanced sensory evidence for threat and the blue arrows represent imprecise priors. OFC, orbital frontal cortex; SN, substantia nigra. 


\section{Prediction error in early visual areas}

The pulvinar, in addition to its role in attention and relevance modulation, is thought to influence the gain of visual information according to the precision of our prior expectations ${ }^{1}$ (see Box 3). Pulvinar neurons encode the certainty of perceptual decisions while also modulating the gain of prediction error units in a myriad of cortical sensory and associative areas ${ }^{1}$. This is an ongoing iterative process ${ }^{44}$ that, presumably, influences sensory information that inputs the pulvinar, such as that received from the SC or via direct retinal afferents. Crucially, the pulvinar modulates the confidence of perceptual decisions, which dictates whether we engage in a decision at all ${ }^{117}$. Pulvinar inhibition reduces confidence and results in more frequent decisions to 'opt-out'. Confidence has been suggested to arise from a sharpening of prediction error signals (that is, increasing their precision and neural 'gain' of the signal) ${ }^{1}$, which, in turn, is modulated by attention ${ }^{118}$. Accordingly, the pulvinar is in a position to more precisely encode attended, compared with unattended, stimuli ${ }^{45}$ as well as modulate the impact of attention via cortico-pulvino-cortical loops ${ }^{44}$.

Responses in the SC are also sensitive to prior expectation. Neurons within the SC respond to novel stimuli, evoking faster saccade latencies for more surprising stimuli ${ }^{119}$. These SC responses to unpredictable and salient events are transmitted rapidly and directly to dopaminergic neurons within the substantia nigra ${ }^{120}$. Crucially, these rapid stimulus responses, and the behaviours they elicit (for example, anticipatory licking in monkeys), occur even in the absence of V1 (ref. ${ }^{121}$ ). Hence, pathways from the SC to the dopaminergic reward system, via the midbrain, constitute a potential shortcut for predictive reward processing. Interestingly, a follow-up study has demonstrated that, if $\mathrm{V} 1$ remains intact and the SC is inactivated instead, dopaminergic neurons respond just as quickly (although saccades were slower $)^{122}$, despite the path from V1 not being direct ${ }^{123}$. This calls into question whether these two seemingly redundant pathways to dopaminergic neurons are co-activated for the simple visual stimuli used in these experiments, or whether using more complex stimuli might result in differences in the human brain.

\section{Box 3 | The pulvinar and intolerance of uncertainty}

To quote horror fiction author H. P. Lovecraft: 'The oldest and strongest emotion of mankind is fear, and the oldest and strongest kind of fear is fear of the unknown.' Uncertainty is inherently anxiety-provoking, as it indicates that we cannot actively seek potential rewards or avoid losses or threats ${ }^{172}$. Intolerance of uncertainty is a core symptom of many psychiatric disorders, including depression ${ }^{186}$, anxiety ${ }^{187}$, eating disorders ${ }^{188}$ and autism ${ }^{189}$. People with higher intolerance of uncertainty may overestimate the likelihood or severity of negative outcomes ${ }^{190}$, worry about the future in an effort to reduce uncertainty ${ }^{191}$ and avoid risk ${ }^{172}$.

The pulvinar is a likely candidate for how uncertainty increases anxiety. For example, activity in the pulvinar and in associated thalamocortical loops is especially magnified by threat in people with stronger attentional threat biases ${ }^{192}$, social anxiety ${ }^{52}$, specific phobia ${ }^{193}$ and depression ${ }^{194}$. There is also evidence that the pulvinar responds to cues pertaining to negative hypothetical future events ${ }^{195}$. Hence, an intolerance of uncertainty may be the result of the pulvinar 'overfeeding' the areas it connects to (including the amygdala). Specifically, the pulvinar may increase the gain of all incoming stimuli in uncertain conditions (owing to diminished top-down signals from areas such as the dorsomedial prefrontal cortex ${ }^{172}$, similar to how stress sharpens the precision of sensory prediction errors ${ }^{171}$ ), resulting in hypervigilance and reinforcement of negative expectations. There is wide scope for future research to acquire new evidence for or against this proposition.

\section{Threat anticipation}

An emerging pattern in the literature is the relationship between trait anxiety, threat predictability and conscious perception. For example, people with higher trait anxiety are faster to detect fearful faces in breaking continuous flash suppression ${ }^{124}$ and backward masking ${ }^{125}$. Furthermore, in an emotional expression recognition task, expecting to see an upcoming fearful face improved signal detection (that is, fearful faces versus neutral faces) and the response time ${ }^{126,127,128}$. This was especially the case when an anxious state had been induced via anticipation of an upcoming electric shock. These effects interact with participants' trait anxiety, such that people with higher trait anxiety show improved perceptual sensitivity when expecting fearful faces, but not neutral faces, during shock anticipation ${ }^{127}$. Beyond faces, anxiety is also associated with more negative interpretations of ambiguous scenarios and interoceptive, bodily sensations (for a review, see ref. ${ }^{129}$ ). 
At which points in a neural hierarchy does incoming visual information first impinge on a prior expectation for threat? Aversive prediction errors have been observed in the basolateral amygdala ${ }^{130}$, PAG $^{131}$ and striatum ${ }^{132}$. These prediction errors are not generic 'surprise' signals but, rather, encode the specific features of representations that were unexpected, given prior experience ${ }^{133}$. For example, top-down predictions transmitted from the ventromedial prefrontal cortex to the PAG generate prediction errors regarding whether a nocioceptive input received from the body, via the PAG, was more or less painful than expected ${ }^{131}$. As another example, prediction errors generated in $\mathrm{V} 1$ after the unexpected absence of a visual stimulus are orientation-specific ${ }^{134}$. This 'match-to-template' method ${ }^{135}$ emphasizes the quality of sensory representations (and the specificity of the prediction) in dictating the information encoded by prediction errors.

In the context of threat perception and expectations, this knowledge suggests that prediction errors generated by the amygdala are influenced by rapid visual input provided by the SC and the pulvinar in two ways (see Fig. 3). The first is via spatial frequency filtering. The SC and the pulvinar receive a predominantly magnocellular input, enabling fast ${ }^{136}$ and unconscious ${ }^{137}$ responses to the 'coarse' visual properties in stimuli that convey biologically relevant information ${ }^{138}$. Should the amygdala receive this coarser, perceptually ambiguous information earlier than a more refined and perceptually specific visual representation from the cortical visual stream, then the threatbiased expectations seen in anxiety disorders could plausibly have a greater influence on perception. The second way is via selective attention. As discussed above, the SC boosts the gain of sensory signals according to their saliency ${ }^{43}$ (for example, facial expressions ${ }^{41,139}$ ). The pulvinar can then enhance the precision of prediction errors generated by the amygdala and visual cortical stream ${ }^{1}$, leading to exaggerated updating of aversive value seen in disorders, such as PTSD ${ }^{140}$. These two propositions are, of course, yet to be empirically tested, but each has crucial implications for when and how visual input interacts with prior expectations in subcortical-cortical hierarchies ${ }^{141}$.

In addition to sensory information about the external world (such as, visual information, which has been the focus of this Review), the brain also incorporates internal information (that is, the body's physiological state) into the generation and updating of beliefs ${ }^{142}$. This process is known as interoceptive (or embodied) inference. Internal states can significantly bias perception by enhancing the precision of sensory evidence for a particular hypothesis. For example, fearful stimuli are more easily and intensely perceived when presented during the systolic phase of a cardiac cycle (for a review, see ref. ${ }^{143}$ ). This effect co-varies with subjective feelings of anxiety, as well as with the BOLD signal in the amygdala ${ }^{144}$ and PAG $^{145}$. Hence, subcortical visual shortcuts likely modulate fear perception in two interacting ways (see Fig. 3). First, the SC-pulvinar-amygdala pathway may evoke a heightened physiological state through non-conscious processing of fearful stimuli (as demonstrated in blindsight research ${ }^{146}$ ). Second, incoming visual input to the SC and pulvinar may be modulated by interoceptive signals about bodily state, such as those sent from the PAG and locus coeruleus to the SC and pulvinar ${ }^{147,148}$. Indeed, functional connectivity strength between the pulvinar and posterior parietal cortex co-varies with pupil-linked arousal ${ }^{149}$. Together, these form a shortcut loop by which negative bodily states are heightened and bias subsequent visual representations towards threat ${ }^{150}$.

\section{Prediction in schizophrenia and autism}

Perhaps the most extreme case of biased perception occurs in hallucinatory phenomena that characterize schizophrenia. Perceptual experiences of people in the early stages of schizophrenia are reported to be more intense (that is, brighter, louder and so forth), and eventually these lead to hallucinations ${ }^{151}$. Current models of schizophrenia pose both altered sensory processing and extremely distorted prior expectations as explanatory factors for psychosis ${ }^{152}$.

Similar to autism, visual processing deficits in schizophrenia are most prominent in the domain of face and motion perception. The pulvinar has been strongly implicated in both cases, albeit in different ways. One study observed impaired facial expression recognition and signal detection for motion in both schizophrenia and autism ${ }^{153}$. In participants with schizophrenia, these impairments were explained by reduced sensory activation and a reduced pulvinar BOLD signal (the latter also correlated with reduced alpha activity measured with electroencephalography). In contrast, behavioural impairment in participants with autism was explained by 
hypersensitive sensory processing and hyper-connectivity of the pulvinar with $\mathrm{V} 1, \mathrm{~V} 5$ and the dorsal visual stream. These findings corroborate a multitude of evidence for a deterioration of the pulvinar and its connections in schizophrenia ${ }^{154,155}$, and an expansion of the pulvinar in autism ${ }^{156,157}$.

\section{A model of subcortical influence}

\section{Concluding remarks}

In this Review, we present an amalgamation of recent research from humans and other animals that suggests an emerging mechanistic account of rapid sensory information processing that serves a range of adaptive behaviours. We focused on the visual domain, given its sensory dominance in humans. We also focused on threat responses owing to relevance for putative fast-acting neural circuits, and their relation to the broad functions of attention and prediction. The summarized research highlights the explanatory power of early subcortical sensory processing, including how processing 'shortcuts' might contribute to models of phenomena seen in psychiatric disorders. This account may complement cortico-centric models that provide a current dominant perspective ${ }^{158}$.

In essence, subcortical circuits can have profound influences on perceptual experience and decision-making via two mechanisms. The first is via altered computations performed by subcortical areas themselves (for example, enhanced responses to threat in the pulvinar ${ }^{40}$, lower decision thresholds for escape in the $\mathrm{SC}^{18}$ and so forth). These alterations can influence the strength and quality of visual representations by filtering our perceptual experience and, ultimately, changing how and what we learn about the world ${ }^{159}$. The second mechanism is via top-down cortical control over regions that receive sensory input earlier (for example, inhibitory effects of V1 over the $\mathrm{SC}^{33,160}$ ), thus gating, or biasing, information processing by higher-order functions such as attention and prediction.

Alterations to either of these mechanisms can have a subtle but cascading effect on multiple, parallel neural circuits, from physiological responses in the body (which may then influence the subsequent interpretation of stimuli) to the precision of visual representations. This latter effect forms a crucial component of predictive coding accounts of the brain, whereby the relative weighting of sensory evidence against prior expectations is thought to explain numerous psychiatric disorders. We encourage future research to consider these early, subcortical networks in models of predictive processing, as their explanatory power will likely make a significant difference to our understanding of neurological disorders.

We speculate that these subcortical networks serve a combination of redundancy and efficiency mechanisms. For threat processing, redundancy is necessary to ensure intact defensive responses to innate signs of danger. Hence, having multiple pathways to key regions such as the amygdala and PAG is adaptive. This multiplicity, however, also likely optimizes defensive behaviour by streamlining different types of information transfer (for example, early coarse versus later detailed visual representations). Other circuits we have discussed, such as projections from the SC versus $\mathrm{V} 1$ to the dopaminergic substantia nigra ${ }^{122}$, appear to serve indistinguishable functions at comparable times. Hence, different subcortical circuits may be more or less redundant for different functions (for example, threat versus reward computations).

\section{Future directions}

There are substantial gaps in the literature on fast subcortical computation in health and psychiatric disorders, in part due to the challenge of measuring subcortical neural dynamics. For example, there is considerable evidence for a role of the SC in computing decision thresholds (where most of the evidence is from invasive animal recordings), yet the potential influence of this has not been explored in neurological disorders ${ }^{161}$. As another example, experimental evidence for how the pulvinar modulates neural responses to surprise ${ }^{1}$ is lacking, especially with regard to psychiatric disorders that feature intolerance of uncertainty ${ }^{162}$. Further investigation into this fundamental component of the predictive brain will clarify our understanding of maladaptive belief formation and the underpinning aberrant neural shortcuts in psychiatric conditions (Box 4).

An ability to shed light on rapid sensory processing within human subcortical areas has been greatly facilitated by anatomical findings that encompass monkey research through to computational reconstructions of human diffusion 
images $^{9,21,22,50}$, as well as estimates of the latency of dynamic neural activity ${ }^{138,163}$. The growing use of magnetoencephalography has proved particularly impactful, given its high temporal resolution and improved ability to spatially resolve sources of neural activity. The recent development of optically pumped magnetometers, a new generation of wearable magnetoencephalography devices whose sensitivity markedly increases the acuity of source reconstruction ${ }^{164}$, is likely to facilitate additional insights into rapid subcortical-cortical interactions during sensory processing and decision-making.

\section{Box 4 | Outstanding questions and future directions}

- Subcortical shortcuts are thought to ensure efficiency by hastening information critical to survival, yet there is no clarity on whether these pathways are myelinated, a factor that can be more important for speed than the pathway length itself ${ }^{175}$. What are the myelination properties of subcortical pathways, such as the superior colliculus-pulvinar-amygdala path? Is their conduction speed quicker overall than the conduction speed of parallel cortical pathways? These questions have important implications for how this subcortical shortcut fits in temporally with other threat processing networks.

- Therapies harnessing unconscious neural activation have promise for reducing attrition in treating psychological disorders, such as specific phobias ${ }^{196}$. What are the precise neural network dynamics (and their cognitive computations) that underlie effective unconscious therapies? Future research can answer this question by examining which neural networks engaged by unconscious therapies (for example, decoded neurofeedback techniques ${ }^{197}$ ) are predictive of successful treatment outcomes.

- The pulvinar is positioned at the interface between incoming visual information and an array of subcortical and cortical networks that compute affective responses, prior expectations and updating of beliefs ${ }^{1}$. Given that many psychopathologies are defined by an intolerance of uncertainty (for example, anxiety) ${ }^{162}$ and aberrant belief formation ${ }^{198}$, does the pulvinar contribute to psychopathologies involving disordered predictive processing? We encourage a new avenue of research to investigate pulvinar activity and ascribed computations under different expectation conditions, in health and neurological disorders. 


\section{Glossary}

\section{Neuroanatomical tracing}

An invasive neuroimaging technique that involves injecting dye into either the cell body of a neuron (that is, anterograde tracing) or a neural synapse (that is, retrograde tracing) to visualize anatomical projections.

\section{Diffusion imaging}

A variant of MRI that measures the diffusion of water molecules that, in the brain, is restricted by the structure of biological tissue (for example, white matter tracts).

\section{GABAergic}

A description of neurons that use the neurotransmitter GABA (that is, $y$-aminobutryic acid, which reduces neuronal excitability).

\section{Fractional anisotropy}

A measure derived from diffusion-weighted images that describes how restricted the diffusion process was, from 0 (isotropic, unrestricted in all directions) to 1 (anisotropic, restricted to one axis).

\section{Tectopulvinar}

Anatomical features pertaining to the tectum (that is, uppermost part of the midbrain, including the superior colliculus) and the pulvinar.

\section{Geniculostriate}

Anatomical features pertaining to the lateral geniculate nucleus and the striate cortex (that is, the primary visual cortex (V1)).

\section{Saliency maps}

Topographically organized maps of the degree to which a stimulus differs in its sensory properties from its surroundings.

\section{Gabor patches}

Striped circular stimuli that have a particular spatial frequency and orientation, created by convolving a Gaussian kernel with a sinusoidal wave.

\section{Electroencephalography}

A non-invasive functional neuroimaging method that uses scalp electrodes to measure electric activity.

\section{Magnetoencephalography}

A non-invasive functional neuroimaging method that uses sensitive external sensors to measure the magnetic fields emitted by electrical currents within the brain.

\section{Acknowledgements}

R.J.D. and J.M. were supported by the Wellcome Trust (098362/A/12/Z and 091593/Z/10/Z) and M.I.G. by the University of Queensland (2016000071). The authors thank the reviewers for their insightful comments on the 
manuscript, J. B. Mattingley for his helpful discussions and all of the researchers who conducted the experiments discussed in this Review. Finally, they especially thank the late patient T.N., whose generosity and willingness to help has made a significant and lasting impact on our understanding of blindsight in the human brain.

\section{References}

1. Kanai, R., Komura, Y., Shipp, S. \& Friston, K. Cerebral hierarchies: predictive processing, precision and the pulvinar. Philos. Trans. R. Soc. B Biol. Sci. 370, 20140169 (2015). This theoretical paper provides a neurobiological account of how the brain coordinates first-order (that is, perceptual content) and secondorder (that is, modulatory gain control by the pulvinar) neural populations to optimize hierarchical predictive inference.

2. Weierich, M. R. \& Treat, T. A. Mechanisms of visual threat detection in specific phobia. Cogn. Emot. 29, 9921006 (2015).

3. Lake, A. J., Baskin-Sommers, A. R., Li, W., Curtin, J. J. \& Newman, J. P. Evidence for unique threat-processing mechanisms in psychopathic and anxious individuals. Cogn. Affect. Behav. Neurosci. 11, 451-462 (2011).

4. Ledoux, E. \& Reis, J. Subcortical efferent projections of the medial geniculate nucleus mediate emotional responses conditioned to acoustic stimuli. J. Neurosci. 4, 16 (1984).

5. Pessoa, L. \& Adolphs, R. Emotion processing and the amygdala: from a 'low road' to 'many roads' of evaluating biological significance. Nat. Rev. Neurosci. 11, 773-783 (2010). This Review debates against the notion that the pulvinar mediates rapid transmission of threatening information to the amygdala, suggesting instead that the pulvinar's role in affective processing is to coordinate cortical responses.

6. Pessoa, L. \& Adolphs, R. Emotion and the brain: multiple roads are better than one. Nat. Rev. Neurosci. 12, 425 (2011).

7. Tamietto, M. \& De Gelder, B. Neural bases of the non-conscious perception of emotional signals. Nat. Rev. Neurosci. 11, 697 (2010). This Review discusses evidence for non-conscious affective processing being facilitated by a pathway from the SC to the amygdala via the pulvinar, the notion of which sparked considerable debate in the literature.

8. de Gelder, B., van Honk, J. \& Tamietto, M. Emotion in the brain: of low roads, high roads and roads less travelled. Nat. Rev. Neurosci. 12, 425 (2011). Together with Pessoa and Adolphs (2010, 2011), this paper debates the notion presented by Tamietto and De Gelder (2010).

9. McFadyen, J., Mattingley, J. B. \& Garrido, M. I. An afferent white matter pathway from the pulvinar to the amygdala facilitates fear recognition. eLife 8, e40766 (2019).

10. Elorette, C., Forcelli, P. A., Saunders, R. C. \& Malkova, L. Colocalization of tectal inputs with amygdalaprojecting neurons in the macaque pulvinar. Front. Neural Circuits 12, 91 (2018). This study is the first to trace a continuous anatomical pathway from the SC to the amygdala, via the pulvinar, in the primate (macaque) brain.

11. Carr, J. A. I'll take the low road: the evolutionary underpinnings of visually triggered fear. Front. Neurosci. 9 , 414 (2015).

12. Yilmaz, M. \& Meister, M. Rapid innate defensive responses of mice to looming visual stimuli. Curr. Biol. 23, 2011-2015 (2013).

13. Vagnoni, E., Lourenco, S. F. \& Longo, M. R. Threat modulates perception of looming visual stimuli. Curr. Biol. 22, R826-R827 (2012). 
14. Wei, P. et al. Processing of visually evoked innate fear by a non-canonical thalamic pathway. Nat. Commun. 6, 6756 (2015).

15. Shang, C. et al. A parvalbumin-positive excitatory visual pathway to trigger fear responses in mice. Science 348, 1472-1477 (2015). This study demonstrates that a pathway from the SC to the parabigeminal nucleus that responds to looming visual stimuli also evokes fearful, defensive behaviour in mice.

16. Zhou, Z. et al. A VTA GABAergic neural circuit mediates visually evoked innate defensive responses. Neuron 103, 1472-1477 (2019).

17. Salay, L. D., Ishiko, N. \& Huberman, A. D. A midline thalamic circuit determines reactions to visual threat. Nature 557, 183 (2018).

18. Evans, D. A. et al. A synaptic threshold mechanism for computing escape decisions. Nature 558,590 (2018). This study demonstrates that the magnitude of neural firing in the SC (in response to a looming visual stimulus) gradually ramps up until a certain threshold, at which a burst of activity in the periaqueductal grey is triggered and the mouse escapes.

19. Ellis, E. M., Gauvain, G., Sivyer, B. \& Murphy, G. J. Shared and distinct retinal input to the mouse superior colliculus and dorsal lateral geniculate nucleus. J. Neurophysiol. 116, 602-610 (2016).

20. Perry, V. H. \& Cowey, A. Retinal ganglion cells that project to the superior colliculus and pretectum in the macaque monkey. Neuroscience 12, 1125-1137 (1984).

21. Rafal, R. D. et al. Connectivity between the superior colliculus and the amygdala in humans and macaque monkeys: virtual dissection with probabilistic DTI tractography. J. Neurophysiol. 114, 1947-1962 (2015).

22. Tamietto, M., Pullens, P., de Gelder, B., Weiskrantz, L. \& Goebel, R. Subcortical connections to human amygdala and changes following destruction of the visual cortex. Curr. Biol. 22, 1449-1455 (2012).

23. Hoy, J. L., Bishop, H. I. \& Niell, C. M. Defined cell types in superior colliculus make distinct contributions to prey capture behavior in the mouse. Curr. Biol. 29, 4130-4138 (2019).

24. Reinhard, K. et al. A projection specific logic to sampling visual inputs in mouse superior colliculus. eLife 8 , e50697 (2019).

25. Shang, C. et al. Divergent midbrain circuits orchestrate escape and freezing responses to looming stimuli in mice. Nat. Commun. 9, 1232 (2018).

26. Vale, R., Evans, D. A. \& Branco, T. Rapid spatial learning controls instinctive defensive behavior in mice. Curr. Biol. 27, 1342-1349 (2017).

27. Liden, W. H., Phillips, M. L. \& Herberholz, J. Neural control of behavioural choice in juvenile crayfish. Proc. R. Soc. B Biol. Sci. 277, 3493-3500 (2010).

28. Evans, D. A., Stempel, A. V., Vale, R. \& Branco, T. Cognitive control of escape behaviour. Trends Cogn. Sci. 23, 334-348 (2019).

29. Keller, G. B. \& Mrsic-Flogel, T. D. Predictive processing: a canonical cortical computation. Neuron 100, 424435 (2018).

30. LeDoux, J. \& Daw, N. D. Surviving threats: neural circuit and computational implications of a new taxonomy of defensive behaviour. Nat. Rev. Neurosci. 19, 269 (2018).

31. Almada, R. C. et al. Stimulation of the nigrotectal pathway at the level of the superior colliculus reduces threat recognition and causes a shift from avoidance to approach behavior. Front. Neural Circuits 12, 36 (2018). 
32. Comoli, E. et al. Segregated anatomical input to sub-regions of the rodent superior colliculus associated with approach and defense. Front. Neuroanat. 6, 9 (2012).

33. Liang, F. et al. Sensory cortical control of a visually induced arrest behavior via corticotectal projections. Neuron 86, 755-767 (2015).

34. Liu, X. et al. Gentle handling attenuates innate defensive responses to visual threats. Front. Behav. Neurosci. 12, 239 (2018).

35. DesJardin, J. T. et al. Defense-like behaviors evoked by pharmacological disinhibition of the superior colliculus in the primate. J. Neurosci. 33, 150-155 (2013).

36. Forcelli, P. A. et al. Amygdala selectively modulates defensive responses evoked from the superior colliculus in non-human primates. Soc. Cogn. Affect. Neurosci. 11, 2009-2019 (2016).

37. Forcelli, P. A., Waguespack, H. F. \& Malkova, L. Defensive vocalizations and motor asymmetry triggered by disinhibition of the periaqueductal gray in non-human primates. Front. Neurosci. 11, 163 (2017).

38. Gandhi, N. J. \& Katnani, H. A. Motor functions of the superior colliculus. Annu. Rev. Neurosci. 34, 205-231 (2011).

39. Bridge, H., Leopold, D. A. \& Bourne, J. A. Adaptive pulvinar circuitry supports visual cognition. Trends Cogn. Sci. 20, 146-157 (2016). This Review discusses evidence from developmental neuroscience for the pulvinar as a higher-order thalamic nucleus that has a fundamental role in coordinating and modulating broad cognitive functions across the brain.

40. Le, Q. V. et al. Monkey pulvinar neurons fire differentially to snake postures. PLoS One 9, e114258 (2014).

41. Nguyen, M. N. et al. Neuronal responses to face-like and facial stimuli in the monkey superior colliculus. Front. Behav. Neurosci. 8, 85 (2014).

42. Maior, R. S., Hori, E., Tomaz, C., Ono, T. \& Nishijo, H. The monkey pulvinar neurons differentially respond to emotional expressions of human faces. Behav. Brain Res. 215, 129-135 (2010).

43. Krauzlis, R. J., Lovejoy, L. P. \& Zénon, A. Superior colliculus and visual spatial attention. Annu. Rev. Neurosci. 36, 165-182 (2013).

44. Saalmann, Y. B., Pinsk, M. A., Wang, L., Li, X. \& Kastner, S. The pulvinar regulates information transmission between cortical areas based on attention demands. Science 337, 753-756 (2012).

45. Fischer, J. \& Whitney, D. Attention gates visual coding in the human pulvinar. Nat. Commun. 3, 1051 (2012).

46. Wise, T., Michely, J., Dayan, P. \& Dolan, R. J. A computational account of threat-related attentional bias. PLoS Comput. Biol. 15, e1007341 (2019).

47. Armstrong, T. \& Olatunji, B. O. Eye tracking of attention in the affective disorders: a meta-analytic review and synthesis. Clin. Psychol. Rev. 32, 704-723 (2012).

48. Basanovic, J., Dean, L., Riskind, J. H. \& MacLeod, C. High spider-fearful and low spider-fearful individuals differentially perceive the speed of approaching, but not receding, spider stimuli. Cogn. Ther. Res. 43, 514-521 (2019).

49. Shiban, Y. et al. Treatment effect on biases in size estimation in spider phobia. Biol. Psychol. 121, 146-152 (2016).

50. Koller, K., Rafal, R. D., Platt, A. \& Mitchell, N. D. Orienting toward threat: contributions of a subcortical pathway transmitting retinal afferents to the amygdala via the superior colliculus and pulvinar. Neuropsychologia 128, 7886 (2019). 
51. Nakataki, M. et al. Glucocorticoid administration improves aberrant fear-processing networks in spider phobia. Neuropsychopharmacology 42, 485-494 (2017).

52. Tadayonnejad, R., Klumpp, H., Ajilore, O., Leow, A. \& Phan, K. L. Aberrant pulvinar effective connectivity in generalized social anxiety disorder. Medicine 95, e5358 (2016).

53. Steuwe, C. et al. Effect of direct eye contact in PTSD related to interpersonal trauma: an fMRI study of activation of an innate alarm system. Soc. Cogn. Affect. Neurosci. 9, 88-97 (2012).

54. Steuwe, C. et al. Effect of direct eye contact in women with PTSD related to interpersonal trauma: psychophysiological interaction analysis of connectivity of an innate alarm system. Psychiatry Res. 232, 162-167 (2015).

55. Nguyen, M. N. et al. Neuronal responses to face-like stimuli in the monkey pulvinar. Eur. J. Neurosci. 37, 3551 (2013).

56. Sawyers, C. et al. The genetic and environmental structure of fear and anxiety in juvenile twins. Am. J. Med. Genet. B Neuropsychiatr. Genet. 180, 204-212 (2019).

57. Hormigo, S., Vega-Flores, G. \& Castro-Alamancos, M. A. Basal ganglia output controls active avoidance behavior. J. Neurosci. 36, 10274-10284 (2016).

58. Cohen, J. D. \& Castro-Alamancos, M. A. Neural correlates of active avoidance behavior in superior colliculus. J. Neurosci. 30, 8502-8511 (2010).

59. Jure, R. Autism pathogenesis: the superior colliculus. Front. Neurosci. 12, 1029 (2018). This Review proposes how the SC is likely a significant contributor towards the genesis and symptoms of autism spectrum disorder.

60. Khalil, R., Tindle, R., Boraud, T., Moustafa, A. A. \& Karim, A. A. Social decision making in autism: on the impact of mirror neurons, motor control, and imitative behaviors. CNS Neurosci. Ther. 24, 669-676 (2018).

61. Quattrocki, E. \& Friston, K. Autism, oxytocin and interoception. Neurosci. Biobehav. Rev. 47, 410-430 (2014).

62. Kleinhans, N. M. et al. fMRI evidence of neural abnormalities in the subcortical face processing system in ASD. Neurolmage 54, 697-704 (2011).

63. Zürcher, N. R. et al. Perception of social cues of danger in autism spectrum disorders. PLoS One 8, e81206 (2013).

64. Hadjikhani, N. et al. Look me in the eyes: constraining gaze in the eye-region provokes abnormally high subcortical activation in autism. Sci. Rep. 7, 3163 (2017).

65. Hu, Y. et al. A translational study on looming-evoked defensive response and the underlying subcortical pathway in autism. Sci. Rep. 7, 14755 (2017).

66. Guy, J., Mottron, L., Berthiaume, C. \& Bertone, A. A developmental perspective of global and local visual perception in autism spectrum disorder. J. Autism Dev. Disord. 49, 2706-2720 (2019).

67. Lomber, S. G. Learning to see the trees before the forest: reversible deactivation of the superior colliculus during learning of local and global visual features. Proc. Natl Acad. Sci. USA 99, 4049-4054 (2002).

68. Feldman, J. I. et al. Audiovisual multisensory integration in individuals with autism spectrum disorder: a systematic review and meta-analysis. Neurosci. Biobehav. Rev. 95, 220-234 (2018).

69. Stein, B. E., Stanford, T. R. \& Rowland, B. A. Development of multisensory integration from the perspective of the individual neuron. Nat. Rev. Neurosci. 15, 520-535 (2014). 
70. Jones, W. \& Klin, A. Attention to eyes is present but in decline in 2-6-month-old infants later diagnosed with autism. Nature 504, 427-431 (2013).

71. Overton, P. G. Collicular dysfunction in attention deficit hyperactivity disorder. Med. Hypotheses 70, 11211127 (2008).

72. Panagiotidi, M., Overton, P. G. \& Stafford, T. Attention-deficit hyperactivity disorder-like traits and distractibility in the visual periphery. Perception 46, 665-678 (2017).

73. Munoz, D. P., Armstrong, I. T., Hampton, K. A. \& Moore, K. D. Altered control of visual fixation and saccadic eye movements in attention-deficit hyperactivity disorder. J. Neurophysiol. 90, 503-514 (2003).

74. Panagiotidi, M., Overton, P. \& Stafford, T. Increased microsaccade rate in individuals with ADHD traits. J. Eye Mov. Res. https://doi.org/10.16910/10.1.6 (2017).

75. Clements, K., Devonshire, I., Reynolds, J. \& Overton, P. Enhanced visual responses in the superior colliculus in an animal model of attention-deficit hyperactivity disorder and their suppression by d-amphetamine. Neuroscience 274, 289-298 (2014).

76. Gowan, J., Coizet, V., Devonshire, I. \& Overton, P. d-Amphetamine depresses visual responses in the rat superior colliculus: a possible mechanism for amphetamine-induced decreases in distractibility. J. Neural Transm. 115, 377-387 (2008).

77. Dommett, E. J., Overton, P. G. \& Greenfield, S. A. Drug therapies for attentional disorders alter the signal-tonoise ratio in the superior colliculus. Neuroscience 164, 1369-1376 (2009).

78. Gaymard, B., François, C., Ploner, C. J., Condy, C. \& Rivaud-Péchoux, S. A direct prefrontotectal tract against distractibility in the human brain. Ann. Neurol. 53, 542-545 (2003).

79. Kim, H. F., Amita, H. \& Hikosaka, O. Indirect pathway of caudal basal ganglia for rejection of valueless visual objects. Neuron 94, 920-930.e3 (2017).

80. Hulst, B. M. van et al. Children with ADHD symptoms show decreased activity in ventral striatum during the anticipation of reward, irrespective of ADHD diagnosis. J. Child. Psychol. Psychiatry 58, 206-214 (2017).

81. Day-Brown, J. D., Wei, H., Chomsung, R. D., Petry, H. M. \& Bickford, M. E. Pulvinar projections to the striatum and amygdala in the tree shrew. Front. Neuroanat. 4, 143 (2010).

82. Ivanov, I. et al. Morphological abnormalities of the thalamus in youths with attention deficit hyperactivity disorder. Am. J. Psychiatry 167, 397-408 (2010).

83. $\mathrm{Li}, \mathrm{X}$. et al. Atypical pulvinar-cortical pathways during sustained attention performance in children with attention-deficit/hyperactivity disorder. J. Am. Acad. Child. Adolesc. Psychiatry 51, 1197-1207.e4 (2012).

84. Xia, S. et al. Thalamic shape and connectivity abnormalities in children with attention-deficit/ hyperactivity disorder. Psychiatry Res. 204, 161-167 (2012).

85. Weiskrantz, L., Warrington, E. K., Sanders, M. \& Marshall, J. Visual capacity in the hemianopic field following a restricted occipital ablation. Brain 97, 709-728 (1974).

86. Pegna, A. J., Khateb, A., Lazeyras, F. \& Seghier, M. L. Discriminating emotional faces without primary visual cortices involves the right amygdala. Nat. Neurosci. 8, 24 (2005).

87. Tamietto, M. et al. Unseen facial and bodily expressions trigger fast emotional reactions. Proc. Natl Acad. Sci. USA 106, 17661-17666 (2009).

88. Gelder, B. de et al. Intact navigation skills after bilateral loss of striate cortex. Curr. Biol. 18, R1128-R1129 (2008). 
89. Koch, C., Massimini, M., Boly, M. \& Tononi, G. Neural correlates of consciousness: progress and problems. Nat. Rev. Neurosci. 17, 307 (2016).

90. Mundinano, I.-C. et al. More than blindsight: case report of a child with extraordinary visual capacity following perinatal bilateral occipital lobe injury. Neuropsychologia 128, 178-186 (2019).

91. Ahmadlou, M., Zweifel, L. S. \& Heimel, J. A. Functional modulation of primary visual cortex by the superior colliculus in the mouse. Nat. Commun. 9, 3895 (2018).

92. Schmid, M. C. et al. Blindsight depends on the lateral geniculate nucleus. Nature 466, 373-377 (2010).

93. Yoshida, M. et al. Residual attention guidance in blindsight monkeys watching complex natural scenes. Curr. Biol. 22, 1429-1434 (2012).

94. Corbetta, M. \& Shulman, G. L. Spatial neglect and attention networks. Annu. Rev. Neurosci. 34, 569-599 (2011).

95. Vaessen, M. J., Saj, A., Lovblad, K.-O., Gschwind, M. \& Vuilleumier, P. Structural white-matter connections mediating distinct behavioral components of spatial neglect in right brain-damaged patients. Cortex 77, 54-68 (2016).

96. Driver, J. \& Mattingley, J. B. Parietal neglect and visual awareness. Nat. Neurosci. 1, 17-22 (1998).

97. Bertini, C., Cecere, R. \& Làdavas, E. Unseen fearful faces facilitate visual discrimination in the intact field. Neuropsychologia 128, 58-64 (2019).

98. De Gelder, B., Morris, J. S. \& Dolan, R. J. Unconscious fear influences emotional awareness of faces and voices. Proc. Natl Acad. Sci. USA 102, 18682-18687 (2005).

99. Bertini, C., Cecere, R. \& Làdavas, E. I am blind, but I "see" fear. Cortex 49, 985-993 (2013).

100. Zhan, M. \& de Gelder, B. Unconscious fearful body perception enhances discrimination of conscious anger expressions under continuous flash suppression. Neuropsychologia 128, 325-331 (2019).

101. Cecere, R., Bertini, C., Maier, M. E. \& Làdavas, E. Unseen fearful faces influence face encoding: evidence from ERPs in hemianopic patients. J. Cogn. Neurosci. 26, 2564-2577 (2014).

102. Vlassova, A., Donkin, C. \& Pearson, J. Unconscious information changes decision accuracy but not confidence. Proc. Natl Acad. Sci. USA 111, 16214-16218 (2014).

103. Barbosa, L. S., Vlassova, A. \& Kouider, S. Prior expectations modulate unconscious evidence accumulation. Conscious. Cogn. 51, 236-242 (2017).

104. Hedger, N., Gray, K. L., Garner, M. \& Adams, W. J. Are visual threats prioritized without awareness? A critical review and meta-analysis involving 3 behavioral paradigms and 2696 observers. Psychol. Bull. 142, 934 (2016).

105. Gayet, S., Paffen, C. L., Belopolsky, A. V., Theeuwes, J. \& Van der Stigchel, S. Visual input signaling threat gains preferential access to awareness in a breaking continuous flash suppression paradigm. Cognition 149, $77-$ $83(2016)$.

106. Etkin, A. et al. Individual differences in trait anxiety predict the response of the basolateral amygdala to unconsciously processed fearful faces. Neuron 44, 1043-1055 (2004).

107. Lipka, J., Miltner, W. H. \& Straube, T. Vigilance for threat interacts with amygdala responses to subliminal threat cues in specific phobia. Biol. Psychiatry 70, 472-478 (2011).

108. Neumeister, P. et al. Specific amygdala response to masked fearful faces in post-traumatic stress relative to other anxiety disorders. Psychol. Med. 48, 1209-1217 (2018). 
109. Sato, W., Kochiyama, T., Uono, S., Yoshimura, S. \& Toichi, M. Neural mechanisms underlying conscious and unconscious gaze-triggered attentional orienting in autism spectrum disorder. Front. Hum. Neurosci. 11, 339 (2017).

110. Madipakkam, A. R., Rothkirch, M., Dziobek, I. \& Sterzer, P. Unconscious avoidance of eye contact in autism spectrum disorder. Sci. Rep. 7, 13378 (2017).

111. Akechi, $\mathrm{H}$. et al. Absence of preferential unconscious processing of eye contact in adolescents with autism spectrum disorder. Autism Res. 7, 590-597 (2014).

112. Hohwy, J. Attention and conscious perception in the hypothesis testing brain. Front. Psychol. 3, 96 (2012).

113. Aue, T. \& Okon-Singer, H. Expectancy biases in fear and anxiety and their link to biases in attention. Clin. Psychol. Rev. 42, 83-95 (2015).

114. Brown, H. \& Friston, K. J. Free-energy and illusions: the cornsweet effect. Front. Psychol. 3, 43 (2012).

115. Pinto, Y., van Gaal, S., de Lange, F. P., Lamme, V. A. \& Seth, A. K. Expectations accelerate entry of visual stimuli into awareness. J. Vis. 15, 13 (2015).

116. de Lange, F. P., Heilbron, M. \& Kok, P. How do expectations shape perception? Trends Cogn. Sci. 22, $764-$ 779 (2018). This Review presents neuroscientific evidence of how prior expectations can influence (that is, suppress, enhance or bias) the content of our conscious experience.

117. Komura, Y., Nikkuni, A., Hirashima, N., Uetake, T. \& Miyamoto, A. Responses of pulvinar neurons reflect a subject's confidence in visual categorization. Nat. Neurosci. 16, 749 (2013).

118. Jiang, J., Summerfield, C. \& Egner, T. Attention sharpens the distinction between expected and unexpected percepts in the visual brain. J. Neurosci. 33, 18438-18447 (2013).

119. Basso, M. A. \& Wurtz, R. H. Modulation of neuronal activity in superior colliculus by changes in target probability. J. Neurosci. 18, 7519-7534 (1998).

120. Comoli, E. et al. A direct projection from superior colliculus to substantia nigra for detecting salient visual events. Nat. Neurosci. 6, 974 (2003).

121. Takakuwa, N., Kato, R., Redgrave, P. \& Isa, T. Emergence of visually-evoked reward expectation signals in dopamine neurons via the superior colliculus in V1 lesioned monkeys. eLife 6, e24459 (2017).

122. Takakuwa, N., Redgrave, P. \& Isa, T. Cortical visual processing evokes short-latency reward-predicting cue responses in primate midbrain dopamine neurons. Sci. Rep. 8, 14984 (2018).

123. May, P. J. et al. Tectonigral projections in the primate: a pathway for pre-attentive sensory input to midbrain dopaminergic neurons. Eur. J. Neurosci. 29, 575-587 (2009).

124. Capitão, L. P. et al. Anxiety increases breakthrough of threat stimuli in continuous flash suppression. Emotion 14, 1027 (2014).

125. Damjanovic, L., Meyer, M. \& Sepulveda, F. Raising the alarm: individual differences in the perceptual awareness of masked facial expressions. Brain Cogn. 114, 1-10 (2017).

126. Sussman, T. J., Weinberg, A., Szekely, A., Hajcak, G. \& Mohanty, A. Here comes trouble: prestimulus brain activity predicts enhanced perception of threat. Cereb. Cortex 27, 2695-2707 (2016).

127. Sussman, T. J., Szekely, A., Hajcak, G. \& Mohanty, A. It's all in the anticipation: how perception of threat is enhanced in anxiety. Emotion 16, 320 (2016). 
128. Imbriano, G., Sussman, T. J., Jin, J. \& Mohanty, A. The role of imagery in threat-related perceptual decision making. Emotion https://doi.org/10.1037/ emo0000610 (2019).

129. Hirsch, C. R., Meeten, F., Krahé, C. \& Reeder, C. Resolving ambiguity in emotional disorders: the nature and role of interpretation biases. Annu. Rev. Clin. Psychol. 12, 281-305 (2016).

130. McHugh, S. B. et al. Aversive prediction error signals in the amygdala. J. Neurosci. 34, 9024-9033 (2014).

131. Roy, M. et al. Representation of aversive prediction errors in the human periaqueductal gray. Nat. Neurosci. 17, 1607 (2014).

132. Robinson, O. J., Overstreet, C., Charney, D. R., Vytal, K. \& Grillon, C. Stress increases aversive prediction error signal in the ventral striatum. Proc. Natl Acad. Sci. USA 110, 4129-4133 (2013).

133. Den Ouden, H. E., Kok, P. \& De Lange, F. P. How prediction errors shape perception, attention, and motivation. Front. Psychol. 3, 548 (2012).

134. Kok, P., Failing, M. F. \& de Lange, F. P. Prior expectations evoke stimulus templates in the primary visual cortex. J. Cogn. Neurosci. 26, 1546-1554 (2014).

135. Kok, P., Mostert, P. \& De Lange, F. P. Prior expectations induce prestimulus sensory templates. Proc. Natl Acad. Sci. USA 114, 10473-10478 (2017).

136. Méndez-Bértolo, C. et al. A fast pathway for fear in human amygdala. Nat. Neurosci. 19, 1041 (2016).

137. Burra, N., Hervais-Adelman, A., Celeghin, A., de Gelder, B. \& Pegna, A. J. Affective blindsight relies on low spatial frequencies. Neuropsychologia 128, 44-49 (2019).

138. McFadyen, J., Mermillod, M., Mattingley, J. B., Halász, V. \& Garrido, M. I. A rapid subcortical amygdala route for faces irrespective of spatial frequency and emotion. J. Neurosci. 37, 3864-3874 (2017).

139. Van Le, Q. et al. Gamma oscillations in the superior colliculus and pulvinar in response to faces support discrimination performance in monkeys. Neuropsychologia 128, 87-95 (2019).

140. Homan, P. et al. Neural computations of threat in the aftermath of combat trauma. Nat. Neurosci. 22, 470 (2019).

141. Summerfield, C. \& De Lange, F. P. Expectation in perceptual decision making: neural and computational mechanisms. Nat. Rev. Neurosci. 15, 745 (2014).

142. Pezzulo, G. Why do you fear the bogeyman? An embodied predictive coding model of perceptual inference. Cogn. Affect. Behav. Neurosci. 14, 902-911 (2014).

143. Critchley, H. D. \& Garfinkel, S. N. Interoception and emotion. Curr. Opin. Psychol. 17, 7-14 (2017).

144. Garfinkel, S. N. et al. Fear from the heart: sensitivity to fear stimuli depends on individual heartbeats. J. Neurosci. 34, 6573-6582 (2014).

145. Gray, M. A. et al. Emotional appraisal is influenced by cardiac afferent information. Emotion 12, 180 (2012).

146. Celeghin, A., de Gelder, B. \& Tamietto, M. From affective blindsight to emotional consciousness. Conscious. Cogn. 36, 414-425 (2015).

147. Spreafico, R., Kirk, C., Franceschetti, S. \& Avanzini, G. Brain stem projections to the pulvinar-lateralis posterior complex of the cat. Exp. Brain Res. 40, 209-220 (1980).

148. Edwards, S. B., Ginsburgh, C. L., Henkel, C. K. \& Stein, B. E. Sources of subcortical projections to the superior colliculus in the cat. J. Comp. Neurol. 184, 309-329 (1979). 
149. Stitt, I., Zhou, Z. C., Radtke-Schuller, S. \& Fröhlich, F. Arousal dependent modulation of thalamo-cortical functional interaction. Nat. Commun. 9, 1-13 (2018).

150. Miller, M. \& Clark, A. Happily entangled: prediction, emotion, and the embodied mind. Synthese 195, 25592575 (2018).

151. Horga, G. \& Abi-Dargham, A. An integrative framework for perceptual disturbances in psychosis. Nat. Rev. Neurosci. 20, 763-778 (2019).

152. Lanillos, P. et al. A review on neural network models of schizophrenia and autism spectrum disorder. Neural Netw. 122, 338-363 (2020).

153. Martínez, A. et al. Differential patterns of visual sensory alteration underlying face emotion recognition impairment and motion perception deficits in schizophrenia and autism spectrum disorders. Biol. Psychiatry 86 , 557-567 (2019).

154. Cho, K. I. K. et al. Microstructural changes in higher-order nuclei of the thalamus in patients with first-episode psychosis. Biol. Psychiatry 85, 70-78 (2019).

155. Dorph-Petersen, K.-A. \& Lewis, D. A. Postmortem structural studies of the thalamus in schizophrenia. Schizophr. Res. 180, 28-35 (2017).

156. Schuetze, M. et al. Morphological alterations in the thalamus, striatum, and pallidum in autism spectrum disorder. Neuropsychopharmacology 41, 2627 (2016).

157. Woodward, N. D., Giraldo-Chica, M., Rogers, B. \& Cascio, C. J. Thalamocortical dysconnectivity in autism spectrum disorder: an analysis of the autism brain imaging data exchange. Biol. Psychiatry Cogn. Neurosci. Neuroimaging 2, 76-84 (2017).

158. Lecciso, F. \& Colombo, B. Beyond the cortico-centric models of cognition: the role of subcortical functioning in neurodevelopmental disorders. Front. Psychol. 10, 2809 (2019).

159. Niv, Y. Learning task-state representations. Nat. Neurosci. 22, 1544-1553 (2019).

160. Ahmadlou, M., Tafreshiha, A. \& Heimel, J. A. Visual cortex limits pop-out in the superior colliculus of awake mice. Cereb. Cortex 27, 5772-5783 (2017).

161. Mandali, A., Weidacker, K., Kim, S.-G. \& Voon, V. The ease and sureness of a decision: evidence accumulation of conflict and uncertainty. Brain 142, 1471-1482 (2019).

162. Pepperdine, E., Lomax, C. \& Freeston, M. H. Disentangling intolerance of uncertainty and threat appraisal in everyday situations. J. Anxiety Disord. 57, 31-38 (2018).

163. Garvert, M. M., Friston, K. J., Dolan, R. J. \& Garrido, M. I. Subcortical amygdala pathways enable rapid face processing. Neurolmage 102, 309-316 (2014).

164. Boto, E. et al. A new generation of magnetoencephalography: room temperature measurements using optically-pumped magnetometers. Neurolmage 149, 404-414 (2017).

165. Yu, H.-H., Atapour, N., Chaplin, T. A., Worthy, K. H. \& Rosa, M. G. P. Robust visual responses and normal retinotopy in primate lateral geniculate nucleus following long-term lesions of striate cortex. J. Neurosci. 38, 39553970 (2018).

166. Cerkevich, C. M., Lyon, D. C., Balaram, P. \& Kaas, J. H. Distribution of cortical neurons projecting to the superior colliculus in macaque monkeys. Eye Brain 6, 121-137 (2014).

167. Berman, R. A., Joiner, W. M., Cavanaugh, J. \& Wurtz, R. H. Modulation of presaccadic activity in the frontal eye field by the superior colliculus. J. Neurophysiol. 101, 2934-2942 (2009). 
168. Doubell, T. P., Skaliora, I., Baron, J. \& King, A. J. Functional connectivity between the superficial and deeper layers of the superior colliculus: an anatomical substrate for sensorimotor integration. J. Neurosci. 23, 6596-6607 (2003).

169. White, B. J., Kan, J. Y., Levy, R., Itti, L. \& Munoz, D. P. Superior colliculus encodes visual saliency before the primary visual cortex. Proc. Natl Acad. Sci. USA 114, 9451-9456 (2017). This study demonstrates, using simultaneous recordings of the SC and V1 in Rhesus monkeys, that differential responses to saliency occurred in the SC before V1.

170. Bisley, J. W. \& Mirpour, K. The neural instantiation of a priority map. Curr. Opin. Psychol. 29, 108-112 (2019).

171. Peters, A., McEwen, B. S. \& Friston, K. Uncertainty and stress: why it causes diseases and how it is mastered by the brain. Prog. Neurobiol. 156, 164-188 (2017).

172. Grupe, D. W. \& Nitschke, J. B. Uncertainty and anticipation in anxiety: an integrated neurobiological and psychological perspective. Nat. Rev. Neurosci. 14, 488-501 (2013).

173. Maunsell, J. H. \& Gibson, J. R. Visual response latencies in striate cortex of the macaque monkey. J. Neurophysiol. 68, 1332-1344 (1992).

174. Yan, Y., Zhaoping, L. \& Li, W. Bottom-up saliency and top-down learning in the primary visual cortex of monkeys. Proc. Natl Acad. Sci. USA 115, 10499-10504 (2018).

175. Schmolesky, M. T. et al. Signal timing across the macaque visual system. J. Neurophysiol. 79, 3272-3278 (1998).

176. Bell, A. H., Meredith, M. A., Van Opstal, A. J. \& Munoz, D. P. Stimulus intensity modifies saccadic reaction time and visual response latency in the superior colliculus. Exp. Brain Res. 174, 53-59 (2006).

177. Silverstein, D. N. \& Ingvar, M. A multi-pathway hypothesis for human visual fear signaling. Front. Syst. Neurosci. 9, 101 (2015).

178. Zeki, S. Area V5 - a microcosm of the visual brain. Front. Integr. Neurosci. 9, 21 (2015).

179. Celeghin, A., Bagnis, A., Diano, M. \& Méndez, C. A. Functional neuroanatomy of blindsight revealed by activation likelihood estimation meta-analysis. Neuropsychologia 128, 109-118 (2019).

180. Ajina, S. \& Bridge, H. Subcortical pathways to extrastriate visual cortex underlie residual vision following bilateral damage to V1. Neuropsychologia 128, 140-149 (2019).

181. Ajina, S. \& Bridge, H. Blindsight relies on a functional connection between hMT+ and the lateral geniculate nucleus, not the pulvinar. PLoS Biol. 16, e2005769 (2018).

182. Tran, A. et al. Neuronal mechanisms of motion detection underlying blindsight assessed by functional magnetic resonance imaging (fMRI). Neuropsychologia 128, 187-197 (2019).

183. Barleben, M. et al. Neural correlates of visual motion processing without awareness in patients with striate cortex and pulvinar lesions. Hum. Brain Mapp. 36, 1585-1594 (2015).

184. Kinoshita, M. et al. Dissecting the circuit for blindsight to reveal the critical role of pulvinar and superior colliculus. Nat. Commun. 10, 135 (2019).

185. Tamietto, M. \& Morrone, M. C. Visual plasticity: blindsight bridges anatomy and function in the visual system. Curr. Biol. 26, R70-R73 (2016).

186. Saulnier, K. G., Allan, N. P., Raines, A. M. \& Schmidt, N. B. Depression and intolerance of uncertainty: relations between uncertainty subfactors and depression dimensions. Psychiatry 82, 72-79 (2018). 
187. Malivoire, B. L. et al. Look before you leap: the role of negative urgency in appraisals of ambiguous and unambiguous scenarios in individuals high in generalized anxiety disorder symptoms. Cogn. Behav. Ther. 48, 217-240 (2018).

188. Brown, M. et al. Intolerance of uncertainty in eating disorders: a systematic review and meta-analysis. Eur. Eat. Disord. Rev. 25, 329-343 (2017).

189. Hodgson, A. R., Freeston, M. H., Honey, E. \& Rodgers, J. Facing the unknown: intolerance of uncertainty in children with autism spectrum disorder. J. Appl. Res. Intellect. Disabil. 30, 336-344 (2017).

190. Paulus, M. P. \& Yu, A. J. Emotion and decision-making: affect-driven belief systems in anxiety and depression. Trends Cogn. Sci. 16, 476-483 (2012).

191. Ranney, R. M., Behar, E. \& Bartoszek, G. Individuals intolerant of uncertainty: the maintenance of worry and distress despite reduced uncertainty. Behav. Ther. 50, 489-503 (2019).

192. Hakamata, Y. et al. The functional activity and effective connectivity of pulvinar are modulated by individual differences in threat-related attentional bias. Sci. Rep. 6, 34777 (2016).

193. Ipser, J. C., Singh, L. \& Stein, D. J. Meta-analysis of functional brain imaging in specific phobia. Psychiatry Clin. Neurosci. 67, 311-322 (2013).

194. Kraus, C. et al. The pulvinar nucleus and antidepressant treatment: dynamic modeling of antidepressant response and remission with ultra-high field functional MRI. Mol. Psychiatry 24, 746-756 (2019).

195. Szpunar, K. K., Jing, H. G., Benoit, R. G., Schacter, D. L. \& Watanabe, K. Repetition-related reductions in neural activity during emotional simulations of future events. PLoS One 10, e0138354 (2015).

196. Taschereau-Dumouchel, V., Liu, K.-Y. \& Lau, H. Unconscious psychological treatments for physiological survival circuits. Curr. Opin. Behav. Sci. 24, 62-68 (2018).

197. Taschereau-Dumouchel, V. et al. Towards an unconscious neural reinforcement intervention for common fears. Proc. Natl Acad. Sci. USA 115, 3470-3475 (2018).

198. Adams, R. A., Huys, Q. J. M. \& Roiser, J. P. Computational psychiatry: towards a mathematically informed understanding of mental illness. J. Neurol. Neurosurg. Psychiatry 87, 53-63 (2016). 